\title{
Averaging of fish target strength functions
}

\author{
Kenneth G. Foote \\ Department of Applied Mathematics, University of Bergen, 5014 Bergen, Norway \\ (Received 12 December 1978; accepted for publication 6 October 1979)

\begin{abstract}
A general model for averaging the acoustic target strength functions of fish is stated in calculable form. It accounts for the influences of the distribution of generally coupled spatial and orientation states of fish, geometric perspective, and beam patterns on observations of target strength. The model is developed and applied to observation of fish by directional, downward-looking sonars. A particular example is considered in which the sonar is represented by an. ideal circular piston, the spatial distribution of fish is homogeneous, and the orientation distribution is spatially homogeneous and characterized by a uniformily distributed azimuthal variable and an independent, essentially normally distributed tilt angle variable. Averaged and averaged-squared backscattering cross sections are computed from high quality gadoid target strength functions measured at two ultrasonic frequencies. Results for a sonar halfbeamwidth of $2.5 \mathrm{deg}$ for three different realizations of the tilt angle distribution are expressed in the logarithmic domain and regressed linearly on fish length. The significance of species, frequency, and orientation distribution differences among the regressions is noted. Estimates of the mean ratio of averaged-squared backscattering cross section and squared-averaged backscattering cross section are presented.
\end{abstract}

PACS numbers: 43.30.Dr, 43.30.Gv, 43.30.Vh, 43.80.Jz

\section{INTRODUCTION}

Quantities of considerable interest in the estimation of the low-order statistics of the acoustic echo energy from an aggregation of fish are the mean and meansquared backscattering cross sections. ${ }^{1-3}$ Computations of averaged backscattering cross sections are reported in the literature. ${ }^{1,4-8}$ These suffer from one or more shortcomings in addition to that of neglect of the geometric effect of perspective. This is visualized simply as the change in apparent target orientation, thence backscattering cross section in general, due to a simple parallel translation of observation point or target. ${ }^{9}$ Because of the perspectival effect, the transmit and receive beam patterns of observing echo sounder cannot be ignored in considering the spatial average of the target strength function, as they might in a dual-beam system. ${ }^{10,11}$ As the systems under review and investigation here are strictly single beam, the averaging operations of Refs. 1 and 4-8 suffer from the neglect of beam patterns. Other shortcomings in averaging of target strengths include neglect of the detailed scattering properties of fish and disregard of fish orientation or distribution of orientation states of fish under observation. There seems to be only a single estimate of the averaged-squared backscattering cross section reported in the literature, ${ }^{1}$ and this suffers from a number of the omissions described here.

Explanations for both the scarcity and erroneous computations of averaged and averaged-squared backscattering cross section may be inferred from the cited literature. These are the lack of simple analyticity in general expression of target strength functions, the need or desire to maintain an easy tractability in computation, and the lack of a general model for averaging.

The several matters are treated in this paper by statement of a general averaging model, development of it by a natural succession of simplifying assumptions, and presentation of numerical computations based on high quality measured target strength functions for an important geometry of application. The decision made at the outset of this study: to perform all involved computations on a high-speed electronic computer, has precluded over-simplification both in the method of averaging and in the representation of target strength functions. The model and computations consequently are believed to be physically realistic. This presumes that the backscattering properties of living fish in the wild at ultrasonic frequencies are adequately represented by corresponding target strength measurements on properly anesthetized, stunned, or killed specimens, at least to the extent that the principal statistics of backscattering cross section are involved. There is both theoretical and empirical evidence for this, but such is preliminary and afield of the present investigation, so is not considered further.

Averaging of the target strength functions of fish is considered first for an arbitrary configuration or ensemble of states of fish as defined by the joint probability distribution of the random variables describing position and orientation of a single fish. This general averaging method is developed for the important special case in which spatial and orientation states are independent. This case, in turn, is developed for the often realistic situation in which fusiform fish of unexceptional behavior are sensed by a directional, downwardlooking sonar. This case is further extended to situations in which the probability of sensing a fish is independent of its position and azimuthal orientation relative to the sonar and the tilt angle is independent of azimuthal orientation.

Computations of averaged and averaged-squared backscattering cross sections are presented for a distinct realization of this particular case; namely, for a downward-looking echo sounder with identical transmit and receive beam patterns equivalent to that of an ideal circular piston of half-beamwidth $2.5 \mathrm{deg}$. The computations are performed for several different modes of behavior, i.e., for several different fish orientation distributions, according to the operational equivalence of fish behavior and orientation distribution defined by Foote. ${ }^{12}$ The target strength functions of application are 
the dorsal aspect target strength functions of a number of gadoid specimens measured at 38 and $120 \mathrm{kHz}$ by Nakken and Olsen ${ }^{4}$ and edited in Ref. 13. Results of computations are expressed through scatter diagrams of averaged target strengths, or root-mean-square target strengths, on fish length or through corresponding least-mean-squares regressions. These computations represent both a continuation and extension of earlier computations. ${ }^{12}$

\section{AVERAGING METHODS}

In the description of averaging methods presented below these conventions are adopted: $F .(\cdot)$ denotes the cumulative distribution or c.d.f. of the variable(s) designated by the subscript(s) when evaluated at the respective argument(s), which may include vector quantities. $d F .\left({ }^{\cdot}\right)$ denotes the probability element or p.e. corresponding to $F .(\cdot) . b^{2}(\hat{k})$ represents the product of transmit and receive beam patterns, which are generally different, when evaluated in the scatterer direction $\hat{k}$. $\langle\sigma\rangle$ denotes the result of averaging the backscattering cross section with respect to the transmit and receive beam patterns and joint distribution of spatial and orientation states, which generally are coupled. $\bar{\sigma}$ denotes an intermediate or partially averaged quantity in those cases where it is possible to separate or decouple at least partly the spatial and orientation states.

The backscattering cross section $\sigma$ of a fish of definite biological and physical condition or state, for a given ensonfication signal, is solely a function of the apparent fish or scatterer orientation as viewed from the observation point at the center of collocated transmitter and receiver. In order to average the backscattering function it is convenient to describe the apparent orientation in terms of the scatterer position and orientation, which provide a more natural description of the scatterer state. Expression of the apparent scatterer orientation in terms of the scatterer orientation and position are facilitated by consideration of the geometry defined in Fig. 1.
Three rectangular coordinate systems are shown. The $(X, Y, Z)$ system is established with its origin at the center of the collocated transmitter and receiver and with the $Z$ axis oriented downwards. The $(x, y, z)$ system is established by simple parallel translation of the $(X, Y, Z)$ system according to the spatial displacement $\mathbf{r}$ which describes the center of rotation of the scatterer relative to the center of collocated transmitter and receiver. The scatterer direction in $(X, Y, Z)$ coordinates is defined by the unit vector $\hat{k}=\mathbf{r} / r$; the observation direction in $(x, y, z)$ coordinates is described by the unit vector $-\hat{k}$. The unit vector $\hat{k}$ is also described by the pair of angles $(\theta, \varphi)$. The $(\xi, \eta, \zeta)$ or scatterer coordinate system is attached to the scatterer at its center of rotation. For a fish the vectors in the positive senses of these axes denote, respectively, frontal, lateral, and ventral aspects, which are described alternatively by normals to the head, side, and belly, as defined with respect to an idealized model of the fish body. The longitudinal axis is described sometimes by the centerline, which is defined as the imaginary line running from the root of the tail to the tip of the upper jaw.

The $(\xi, \eta, \xi)$ system, for an arbitrary scatterer orientation, can be developed from the $(x, y, z)$ system by an ordered succession of three rotations. In the absence of rotation each of the $\xi, \eta, \xi$ axes coincides with the respective axis in the $(x, y, z)$ system. From initial coincidence the scatterer, with attached $(\xi, \eta, \zeta)$ system, is rotated firstly clockwise about the $z$ axis through the angle $\varphi^{\prime}$, which denotes the local azimuthal coordinate or yaw. The scatterer is next rotated clockwise about the lateral body axis, the $\eta$ axis, through the angle $\theta^{\prime}$, which denotes the tilt angle or pitch. The scatterer is finally rotated clockwise about its longitudinal axis, the $\zeta$ axis, through the angle $\psi^{\prime}$, which describes the roll or, as it is alternatively referred to, roll angle.

An arbitrary vector in the $(x, y, z)$ system, say $\mathrm{A}$, is thus described in the $(\xi, \eta, \xi)$ system by the vector $\mathbf{A}^{\prime}=\mathbf{R}^{*} \cdot \mathbf{A}$, where $\mathbf{R}^{*}$ is a unitary matrix with explicit expression

$$
\mathbf{R}^{*}=\left[\begin{array}{ccc}
\cos \theta^{\prime} \cos \varphi^{\prime} & \cos \theta^{\prime} \sin \varphi^{\prime} & -\sin \theta^{\prime} \\
\sin \theta^{\prime} \cos \varphi^{\prime} \sin \psi^{\prime}-\sin \varphi^{\prime} \cos \psi^{\prime} & \sin \theta^{\prime} \sin \varphi^{\prime} \sin \psi^{\prime}+\cos \varphi^{\prime} \cos \psi^{\prime} & \cos \theta^{\prime} \sin \varphi^{\prime} \\
\sin \theta^{\prime} \cos \varphi^{\prime} \cos \psi^{\prime}+\sin \varphi^{\prime} \sin \psi^{\prime} & \sin \theta^{\prime} \sin \varphi^{\prime} \cos \psi^{\prime}-\cos \varphi^{\prime} \sin \psi^{\prime} & \cos \theta^{\prime} \cos \varphi^{\prime}
\end{array}\right]
$$

Thus the apparent scatterer orientation $\hat{\Omega}$ is related to the scatterer direction $\hat{k}$ and scatterer orientation relative to the $(x, y, z)$ system by the equation

$$
\hat{\Omega}=-\mathbf{R}^{*} \cdot \hat{k} \text {. }
$$

The scatterer orientation may also be described by a pair of angles, $\Theta$ and $\Phi$, which, in terms of the rectangular components of $\hat{\Omega}$, the direction cosines $\Omega_{\xi}, \Omega_{\eta}$, and $\Omega_{\zeta}$, are defined by

$$
\theta=\tan ^{-1}\left[\left(\Omega_{\xi}^{2}+\Omega_{\eta}^{2}\right)^{1 / 2} / \Omega_{\xi}\right]
$$

and

$$
\Phi=\tan ^{-1}\left(\Omega_{\eta} / \Omega_{\xi}\right)
$$

The arctangent function is multiple-valued; that branch is chosen for each of $\theta$ and $\Phi$ such that the coordinate pair $(\Theta, \Phi)$ coincides with $(\theta, \varphi)$ for the case in which the scatterer and $(x, y, z)$ coordinate systems coincide, i.e., for the case in which the yaw, pitch, and roll of the scatterer vanish. Thus $(\Theta, \Phi)$ specifies the apparent scatterer orientation when observed from the collocated transmitter and receiver. This interpretation of scatterer orientation is illustrated in Fig. 2.

In expressing the backscattering cross section in 


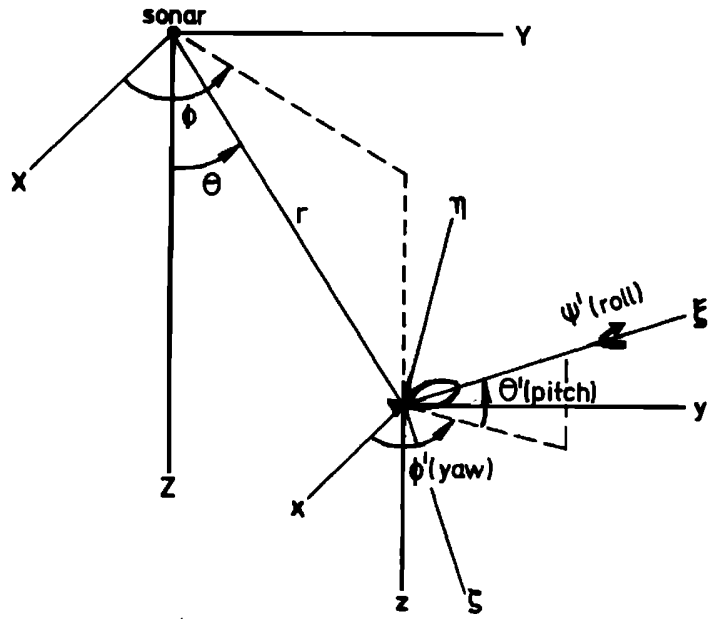

FIG. 1. Geometry of averaging.

averaging operations it is instructive to show the scatterer direction and orientation explicitly in the argument. The scatterer orientation, which is defined by the respective pitch, yaw, and roll angles, $\theta^{\prime}, \varphi^{\prime}$, and $\psi^{\prime}$, will be represented by the symbol $\overline{\mathbf{K}}^{\prime}$. Thus the dependence of $\sigma$ on the apparent scatterer orientation $\hat{\Omega}$; viz. $\sigma=\sigma(\hat{\Omega})$, will be understood although expressed by $\sigma=\sigma\left(\hat{k}, \overline{\mathbf{K}}^{\prime}\right)$.

One further convention is adopted to simplify the expression of averaged quantities: the complexity of arguments is reduced wherever possible. Thus the complexity of arguments of the quantities $b, \sigma$, and $F$ will vary.

The occurrence of a fish in a given position $\mathbf{r}$ with a given orientation $\overline{\mathbf{K}}^{\prime}$ is described by means of the c.d.f. $F_{\mathbf{r}, \tilde{\mathbf{x}}}\left(\mathbf{r}, \overline{\mathbf{K}}^{\prime}\right)$. The spatial and orientation dependence of this function are generally not separable, as in certain avoidance reactions. ${ }^{14-17}$ Because the transmit and receive beam patterns and apparent scatterer orientation are independent of the scatterer distance $r$, the averaging may be performed with respect to the c.d.f. $F_{\hat{k}, \overline{\mathbf{k}}}$, $\left(\hat{k}, \overline{\mathbf{K}}^{\prime}\right)$, which is derived from $F_{\mathbf{r}, \tilde{\mathbf{x}}} \cdot\left(\mathbf{r}, \overline{\mathbf{K}}^{\prime}\right)$ by integration over all possible values of $r$. The averaged cross section may be written

$$
\langle\sigma\rangle=a^{-1} \int b^{2}(\hat{k}) \sigma\left(\hat{k}, \tilde{\mathbf{K}}^{\prime}\right) d F_{\hat{k}, \tilde{\mathbf{K}}}\left(\hat{k}, \overline{\mathbf{K}}^{\prime}\right),
$$

where

$$
a=\int b^{2}(\hat{k}) d F_{\hat{k}}(\hat{k})
$$

specifies the beam pattern normalization. The p.e. $d F_{\hat{k}}(\hat{k})$ is derived from $d F_{k_{,}},\left(\hat{k}, \overline{\mathbf{K}}^{\prime}\right)$ by integ rating over the entire range of scatterer orientation $\tilde{\mathbf{K}}^{\prime}$.

In the absence of certain behavior patterns, such as those manifested by avoidance reactions, the spatial and orientation states of a fish will be independent, i.e., $d F_{\hat{\boldsymbol{k}}, \tilde{\mathbf{\mathbf { x }}}} \cdot\left(\hat{k}, \tilde{\mathbf{K}}^{\prime}\right)=d \boldsymbol{F}_{\hat{k}}(\hat{k}) d \boldsymbol{F}_{\overline{\mathbf{x}}^{\prime}}\left(\overline{\mathbf{K}}^{\prime}\right)$. The mean backscattering cross section may then be expressed by

$$
\langle\sigma\rangle=a^{-1} \int b^{2}(\hat{k}) \bar{\sigma} d F_{\hat{k}}(\hat{k}),
$$

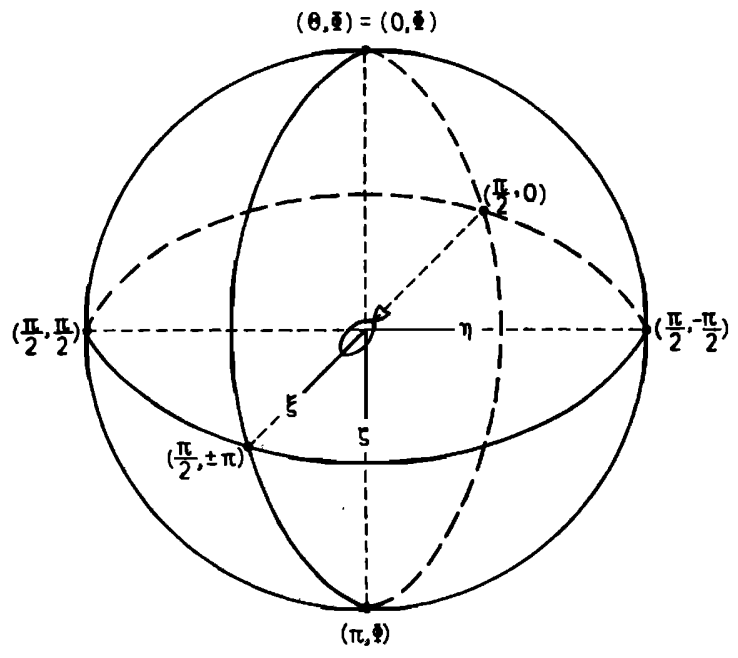

FIG. 2. Description of scatterer orientation in $(\Theta, \Phi)$ coordinates.

where $a$ specifies the beam pattern normalization as in Eq. (6) and

$$
\bar{\sigma}=\int \sigma\left(\hat{k}, \tilde{\mathbf{K}}^{\prime}\right) d F_{\overline{\mathbf{K}}^{\prime}}\left(\tilde{\mathbf{K}}^{\prime}\right)
$$

is the mean backscattering cross section with respect to the ensemble of orientation states for a given scatterer direction.

The orientation distribution-averaged backscattering cross section $\bar{\sigma}$ is now studied for the special and important practical case in which fusiform fish with unexceptional behavior are sensed by a directional, downward-looking echo sounder. In this case the $\psi^{\prime}$ or roll angle dependence of $\sigma$ will be weak for small values of $\theta$ and $\psi^{\prime}$, i.e.,

$$
\sigma\left(\hat{k}, \overline{\mathbf{K}}^{\prime}\right) \approx \sigma\left(\hat{k}, \hat{k}^{\prime}\right)
$$

where $\hat{k}^{\prime}=\left(\cos \theta^{\prime} \cos \varphi^{\prime}, \cos \theta^{\prime} \sin \varphi^{\prime},-\sin \theta^{\prime}\right)$ specifies the orientation of the fish centerline in the $(x, y, z)$ coordinate system. Measurements by Haslett ${ }^{18}$ and Nakken and Olsen, ${ }^{4}$ among others, establish the reasonableness of this approximation under the specified conditions of small roll angles and unexceptional modes of behavior. Because the roll angle dependence of $\sigma$ is trivial, it is ignored below in further statements of the orientation dependence of $\sigma$. Thus

$$
\bar{\sigma}=\int \sigma(\pi / 2-\gamma) d F_{\xi^{\prime}}\left(\hat{k}^{\prime}\right),
$$

where $\pi / 2-\gamma$ specifies the effective tilt angle of the fish centerline, i.e., the apparent tilt angle of the fish as measured from the vantage of the echo sounder;

$$
\gamma=\cos ^{-1}\left[-\sin \theta \cos \theta^{\prime} \cos \left(\varphi-\varphi^{\prime}\right)+\cos \theta \sin \theta^{\prime}\right]
$$

is the supplement of the angle between $\hat{k}$ and $\hat{k}^{\prime}$; and the p.e. $d F_{k^{\prime}}\left(\hat{k}^{\prime}\right)$ is derived from $d F_{\overline{\mathbf{K}} \cdot}\left(\overline{\mathbf{K}}^{\prime}\right)$ by integrating over the entire allowed range of roll angle $\psi^{\prime}$.

A further eminently useful extension of this special case is that for which the probability of observing a fish of any arbitrary tilt angle is independent of its direction $\hat{k}$ and azimuthal orientation $\varphi^{\prime}$ for all positions in or beneath the plane of the echo sounder, i.e., for all $\hat{k} \cdot \hat{z}$ 
TABLE I. Numbers of specimens and length ranges represented by measured dorsal aspect target strength functions used in computations.

\begin{tabular}{|c|c|c|c|c|}
\hline \multirow[b]{2}{*}{ Species } & \multicolumn{2}{|c|}{ Frequency $=38 \mathrm{kHz}$} & \multicolumn{2}{|c|}{ Frequency $=120 \mathrm{kHz}$} \\
\hline & $\begin{array}{l}\text { Number of } \\
\text { specimens }\end{array}$ & $\begin{array}{c}\text { Length range } \\
(\mathrm{cm})\end{array}$ & $\begin{array}{l}\text { Number of } \\
\text { specimens }\end{array}$ & $\begin{array}{l}\text { Length range } \\
\text { (cm) }\end{array}$ \\
\hline $\begin{array}{c}\text { Cod } \\
\text { (Gadus morhua) }\end{array}$ & 68 & $6.7-96.0$ & 44 & $6.7-67.0$ \\
\hline $\begin{array}{c}\text { Saithe } \\
\text { (Pollachius virens) }\end{array}$ & 59 & $9.1-68.0$ & 48 & $9.1-68.0$ \\
\hline $\begin{array}{c}\text { Pollack } \\
\text { (Pollachius pollachius) }\end{array}$ & 44 & $19.7-61.0$ & 39 & $19.7-52.0$ \\
\hline
\end{tabular}

$\geqslant 0$, where $\hat{z}$ is the unit vector in the direction of $Z$ and $z$ axes shown in Fig. 1. Accordingly, the p.e.'s in Eqs. (7) and (10) can be simplified:

$$
\begin{aligned}
& d F_{\hat{k}}(\hat{k})=(2 \pi)^{-1} \sin \theta d \theta d \varphi, \\
& d F_{\hat{k}^{\prime}}\left(\hat{k}^{\prime}\right)=(2 \pi)^{-1} d \varphi^{\prime} d F_{\theta^{\prime}}\left(\theta^{\prime}\right),
\end{aligned}
$$

where $d F_{\theta} \cdot\left(\theta^{\prime}\right)$ is the p.e. of the tilt angle variable.

The assumption of spatial homogeneity contained in Eq. (12) is tantamount to considering the spatial distribution of the fish to be uniform in any observable hemispherical shell centered at and lying beneath the echo sounder. The least and greatest radii of the hemisphere are determined by such practical constraints as the range interval of interest, pulse length, switching time between transmitting and receiving modes if a dual-mode sonar is used, local bottom topography, sound absorption rate, and background noise-adjusted threshhold level of the receiving system.

Those geometries of schooling or aggregating behav ior which are characterized by the grouping of fish in a horizontal layer are not excluded from consideration by the present model. This is because of the assumed directional nature of the downward-looking echo sounder. Only fish located near the acoustic axis can contribute significantly to averages of the backscattering cross section. For sufficiently directional echo sounders the small angle approximation $\sin \theta \doteq \tan \theta \doteq \theta$ is excellent; hence, the applicability of Eq. (12) to the geometries of layered fish distributions in addition to that of a spatially homogeneous distribution.

\section{A COMPUTATIONAL EXAMPLE}

To facilitate the averaging of some dorsal aspect target strength functions of fish for which measurements exist, in order to determine practical measures of the backscattering cross sections of the same fish when observed by a directional, downward-looking sonar beam or echo sounder, a final reduction of the averaging process is now presented.

In this example the transmit and receive beam patterns are assumed identical and equivalent to that produced by an ideal circular piston of half-beamwidth 2.5 deg. The beam pattern thus depends only on the polar angle $\theta$ :

$$
b(\theta)=\left[2 J_{1}(k a \sin \theta) /(k a \sin \theta)\right]^{2},
$$

where the product of wavenumber $k$ and piston radius $a$ is roughly $1.62 / \sin (\pi / 72)=37.1$. This is an excellent approximation for a number of sonars in use on several fisheries research vessels of the Marine Research Institute in Bergen, Norway.

The backscattering cross sections of application are those determined experimentally as a function of tilt angle for a number of gadoid specimens at $\mathbf{3 8}$ and 120 $\mathrm{kHz}$. The measurement of these functions is described in Ref. 4. Some of the data of that study and all of the data considered here are presented in Ref. 13. The numbers of gadoid specimens for which measurements of the dorsal aspect target strength function exist and are used in the computations are shown in Table I together with their corresponding length ranges. Each function consists of measurements of the target strength at one-degree intervals over a $90 \mathrm{deg}$ range in tilt angle centered on the normal horizontal orientation. The values of target strength are expressed in $\mathrm{dB}$ relative to that of an idealized sphere of $2-\mathrm{m}$ radius and perfect geometrical reflectivity. The relation between target strength and backscattering cross section is

$$
\operatorname{TS}\left(\theta^{\prime}\right)=10 \log \left[\sigma\left(\theta^{\prime}\right) / 4 \pi\right] \text {, }
$$

where the tilt angle dependence is shown explicitly. Measurements of the roll angle dependence of the target strength function of a number of specimens by Nakken and Olsen ${ }^{4}$ demonstrated an approximate constancy over a 30 deg range in roll, which, with respect to the directional, downward-looking sonar and application to fish of nonexceptional behavior modes assumed here, justifies neglect of the roll angle dependence in computations involving the target strength. Values of the backscattering cross section for tilt angles lying outside of the measurement range are occasionally needed in averaging operations. These are simulated by the simple unweighted average of $\sigma\left(\theta^{\prime}\right)$ over the nearest $10 \mathrm{deg}$ range of measured values. Such values contribute only slightly to the averages, however, because of their infrequent occurrence.

In the absence of knowledge to the contrary it is most reasonable to assume that the position and orientation of a fish in the sonar beam are independent of the act of observation; in other words, that effects of the kind produced by avoidance reaction, for example, can be ignored. It is similarly reasonable to assume that the azimuthal and tilt angle dependences of the fish orientation are independent. From the photographic observations of Olsen ${ }^{19}$ on free-swimming cod off Lofoten, 
not to mention a priori arguments based on the central limit theorem, the tilt angle distribution of fish may be described by an essentially normal distribution. This observation of normality in tilt angle distribution was confirmed by Beltestad ${ }^{20}$ for the case of free-swimming herring. The precise form of the tilt angle distribution used in the computations is stated in Ref. 12, which follows the uses of two earlier studies. ${ }^{9,21}$. The probability element of tilt angle distribution is $d F_{\theta^{\circ}}\left(\theta^{\prime}\right)=f\left(\theta^{\prime}\right) d \theta^{\prime}$, where the probability density function $f\left(\theta^{\prime}\right)$ is

$$
f\left(\theta^{\prime}\right)=c^{-1} \exp \left[-\left(\theta^{\prime}-\bar{\theta}\right)^{2} / 2 \sigma_{\theta}^{2}\right] \operatorname{rect}\left[\left(\theta^{\prime}-\bar{\theta}\right) / 6 \sigma_{\theta}\right],
$$

where $c$ is the normalization constant,

$$
c=\sigma_{\theta} \int_{-3}^{3} \exp \left(-u^{2} / 2\right) d u \doteq 5 \sigma_{\theta} / 2,
$$

and rect denotes the rectangle function: $\operatorname{rect} x=1$ for $|x| \leqslant \frac{1}{2}$ and 0 for $|x|>\frac{1}{2}$. The two parameters of the distribution which fully define its particular realization are the mean tilt angle $\bar{\theta}$ and the standard deviation $\sigma_{\theta}$ of the nontruncated distribution. The values of $\bar{\theta}$ and $\sigma_{\theta}$ observed by Olsen were approximately -4.4 and $16 \mathrm{deg}$, which almost certainly reflect the behavior of the fish during their observation. In recognition of the behavior dependence of the tilt angle distribution, computations of the mean backscattering cross section are undertaken for this pair of values and two other pairs of values, $(0,5)$ deg and $(0,2) \mathrm{deg}$, which would reflect different modes of behavior. In all cases only rather small values of $\bar{\theta}$ are considered: larger values are regarded as being of unlikely or infrequent occurrence for the gadoids of interest here.

With the beam patterns, geometry of ensonification, target strength functions, and spatial and orientation distribution of individual fish fully specified, the averaged backscattering cross section may now be computed. Because of the axial symmetry of the beam patterns this may be expressed by

$$
\langle\sigma\rangle=a^{-1} \int_{0}^{\tau / 36} b^{2}(\theta) \bar{\sigma} \sin \theta d \theta,
$$

where

$$
a=\int_{0}^{\pi / 36} b^{2}(\theta) \sin \theta d \theta
$$

provides the beam pattern normalization, $b(\theta)$ is defined in Eq. (13), and

$$
\bar{\sigma}=(2 \pi)^{-1} \int_{-\pi / 2}^{\pi / 2} \int_{0}^{2 r} \sigma(\pi / 2-\gamma) d \varphi^{\prime} f\left(\theta^{\prime}\right) d \theta^{\prime},
$$

where $\gamma$ is specified in Eq. (11). The upper limit of integration with respect to $\theta$ is set equal to $\pi / 36 \mathrm{rad}$ or twice the half-beamwidth of $2.5 \mathrm{deg}$. This is an excellent approximation, for

$$
\int_{0}^{\pi / 36} b^{2}(\theta) \sin \theta d \theta \doteq 0.997 \int_{0}^{\pi / 2} b^{2}(\theta) \sin \theta d \theta,
$$

which ensures a computation accuracy better than 0.1 $d B$, which exceeds the accuracy of the basic target strength data. The practical computation of $\bar{\sigma}$ in Eq. (18) may be reduced by recognition of the bilateral symmetry of the fish of application, thence symmetry in target strength function and backscattering cross section with respect to apparent yaw or azimuthal orientation.

The averaged-squared backscattering cross section may be computed by an exactly analogous method. The only difference is that the product of transmit and receive beam patterns and backscattering cross section are replaced by their squares. Thus for the identical conditions of the example the averaged-squared backscattering cross section $\left\langle\sigma^{2}\right\rangle$ is

$$
\left\langle\sigma^{2}\right\rangle=a^{-1} \int_{0}^{\pi / 36} b^{4}(\theta) \overline{\sigma^{2}} \sin \theta d \theta,
$$

where

$$
\begin{aligned}
& a=\int_{0}^{\pi / 36} b^{4}(\theta) \sin \theta d \theta, \\
& \overline{\sigma^{2}}=(2 \pi)^{-1} \int_{\pi / 2}^{\pi / 2} \int_{0}^{2 \pi} \sigma^{2}(\pi / 2-\gamma) d \varphi^{\prime} f\left(\theta^{\prime}\right) d \theta^{\prime},
\end{aligned}
$$

and all other quantities have their previous values.

\section{A. Expression of cross sections}

Because the range of variation in backscattering cross section and in related quantities, such as the averaged and averaged-squared backscattering cross sections; is often large from scatterer to scatterer, it is convenient to use a logarithmic transformation in the presentation of numerical results. With respect to the present investigations averaged and root-mean-square target strengths are defined in terms of $\langle\sigma\rangle$ and $\left\langle\sigma^{2}\right\rangle$ by analogy with Eq. (14). The averaged target strength, denoted $\langle\mathrm{TS}\rangle$, is defined by

$$
\langle\mathrm{TS}\rangle \equiv 10 \log (\langle\sigma\rangle / 4 \pi) \text {. }
$$

The root-mean-square target strength, denoted $\left\langle\mathrm{TS}^{2}\right\rangle^{1 / 2}$, is defined by

$$
\left\langle\mathrm{TS}^{2}\right\rangle^{1 / 2} \equiv 10 \log \left(\left\langle\sigma^{2}\right\rangle^{1 / 2} / 4 \pi\right),
$$

where $\left\langle\sigma^{2}\right\rangle^{1 / 2}$ is used instead of $\left\langle\sigma^{2}\right\rangle$ to facilitate comparison of the several averages.

To emphasize the dependence of the averaged quantities on the orientation distribution of fish and beam patterns of observing sonar, it is occasionally useful to attach subscripts to the averages to denote the values of the characterizing parameters. For the particular computational example considered here it is sufficient to describe three parameters: the two parameters $\vec{\theta}$ and $\sigma_{\theta}$ of the tilt angle distribution and the half-beamwidth $\theta_{3} \mathrm{~dB}$ of the identical axially symmetric transmit and receive beam patterns. Thus $\langle\mathrm{TS}\rangle_{-4.4,16,2.5}$, for example, denotes the averaged target strength corresponding to a backscattering cross section in the mean of the ensemble of orientation states described by the approximately normal tilt angle distribution of Eq. (15) with parameters $\bar{\theta}=-4.4 \mathrm{deg}$ and $\sigma_{\theta}=16 \mathrm{deg}$, for a homogeneous spatial distribution when weighted by identical transmit and receive beam patterns equivalent to that of an ideal circular piston with half-beamwidth $\theta_{3 \mathrm{AB}}=2.5 \mathrm{deg}$. 


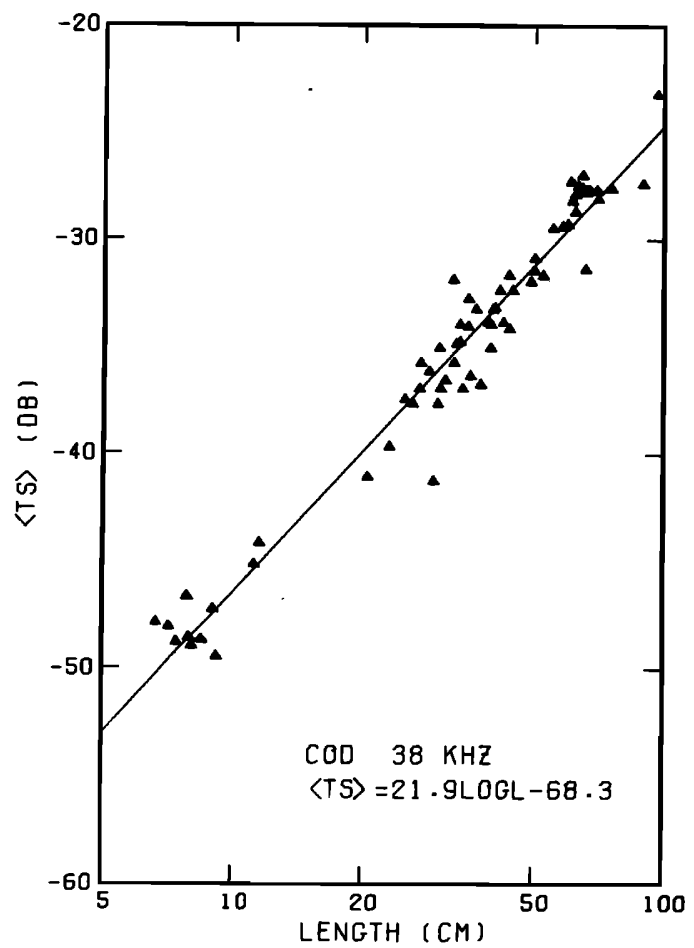

FIG. 3. Scatter diagram and regression of $\langle\mathrm{TS}\rangle-4,4,16,2.5$ on $l$ for cod at $38 \mathrm{kHz}$.

For the concise presentation of the many averages computed here simple linear least-mean squares or minimum variance regression analyses have been carried out for the same quantities when segregated by species and frequency. Both averaged and root-meansquare target strengths have been regressed on fish length $l$, expressed in units of centimeters, according to the equations

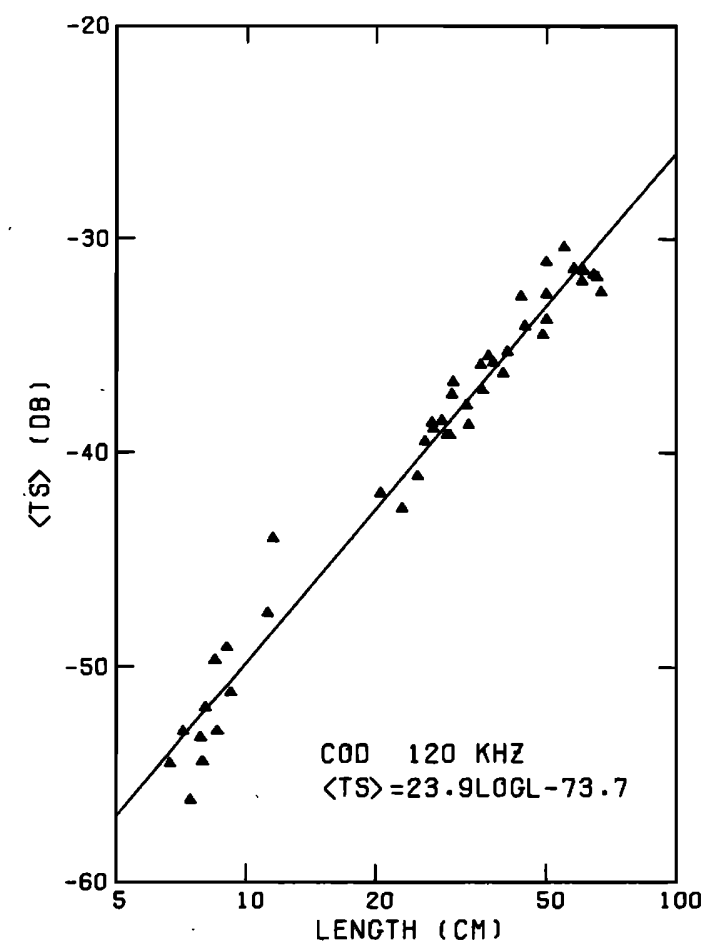

FIG. 4. Scatter diagram and regression of $\langle T S\rangle_{-4.4,16,2.5}$ on $l$ for cod at $120 \mathrm{kHz}$.

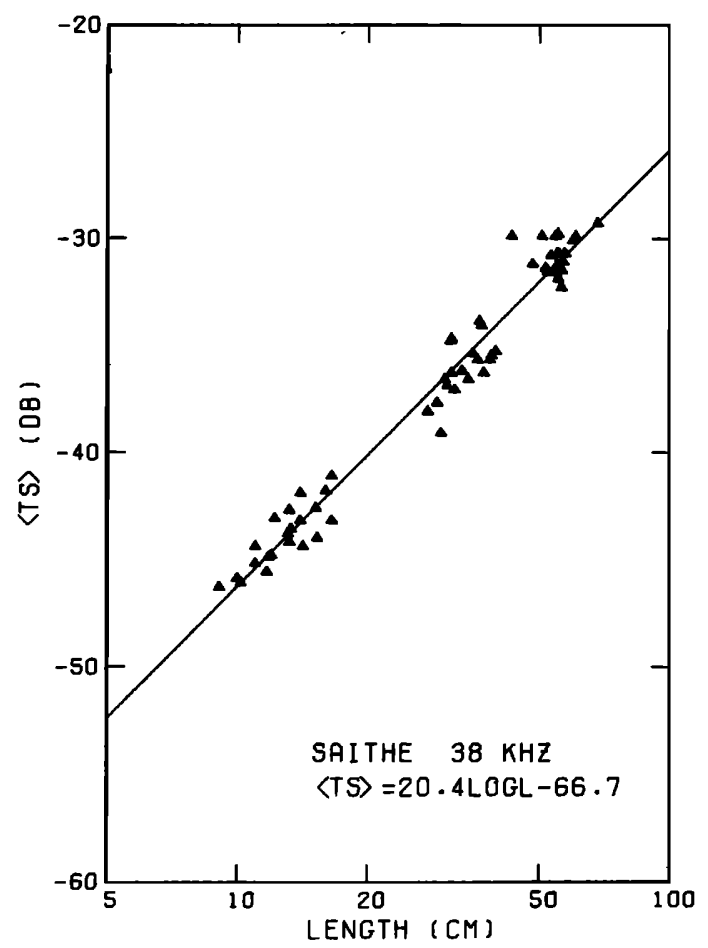

FIG. 5. Scatter diagram and regression of $\langle T S\rangle-4.4,16,2.5$ on $l$ for saithe at $38 \mathrm{kHz}$.

$$
\langle\mathrm{TS}\rangle=\hat{m} \log l+\hat{b}
$$

and

$$
\left\langle\mathrm{TS}^{2}\right\rangle^{1 / 2}=\hat{m} \log l+\hat{b} .
$$

The coefficients $\hat{m}$ and $\hat{b}$ are written with carets to denote their approximate nature as estimates based on a

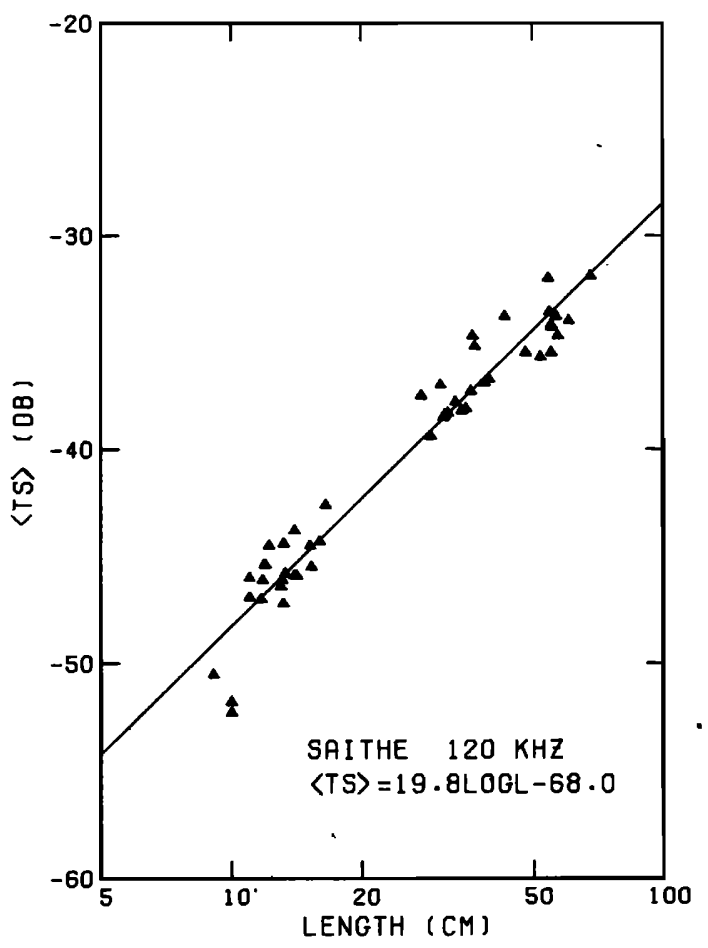

FIG. 6. Scatter diagram and regression of $\langle\mathrm{TS}\rangle-4.4,16,2.5$ on $l$ for saithe at $120 \mathrm{kHz}$.

Kenneth G. Foote: Averaging of fish target strength functions 


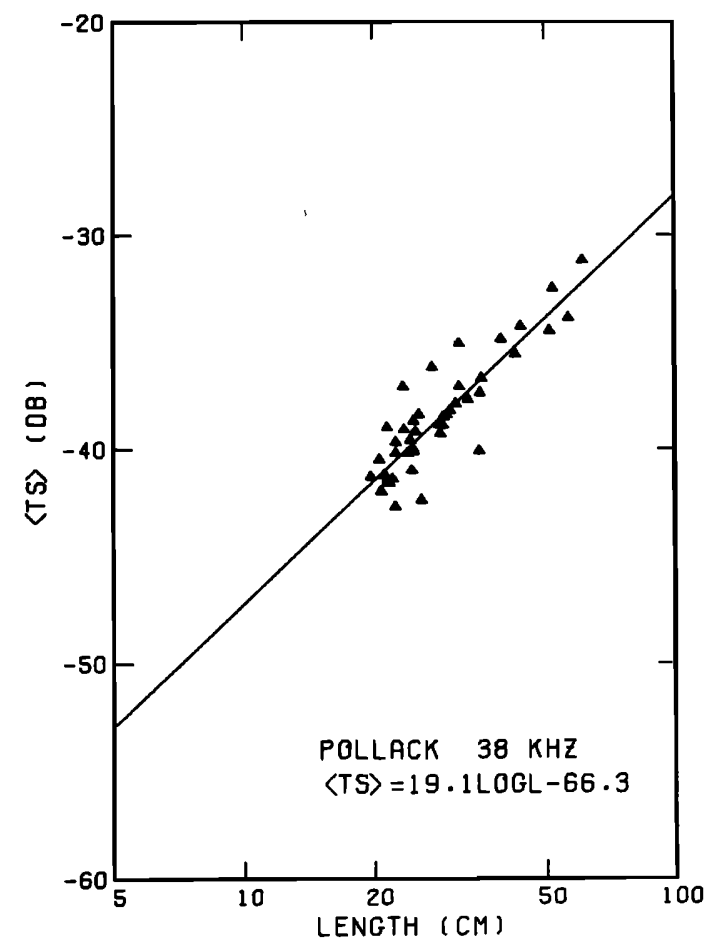

FIG. 7. Scatter diagram and regression of $\langle\mathrm{TS}\rangle_{-4.4,16,2.5}$ on $l$ for pollack at $38 \mathrm{kHz}$.

finite sample size. The estimated standard errors of these coefficients, denoted est $[\mathrm{SE}(\hat{m})]$ and $\operatorname{est}[\mathrm{SE}(\hat{b})]$, as well as the standard error of regression, SE, and correlation coefficient, $\rho$, which are useful quantities in gauging the effectiveness of the linear regression analysis, are computed for each such analysis described below.

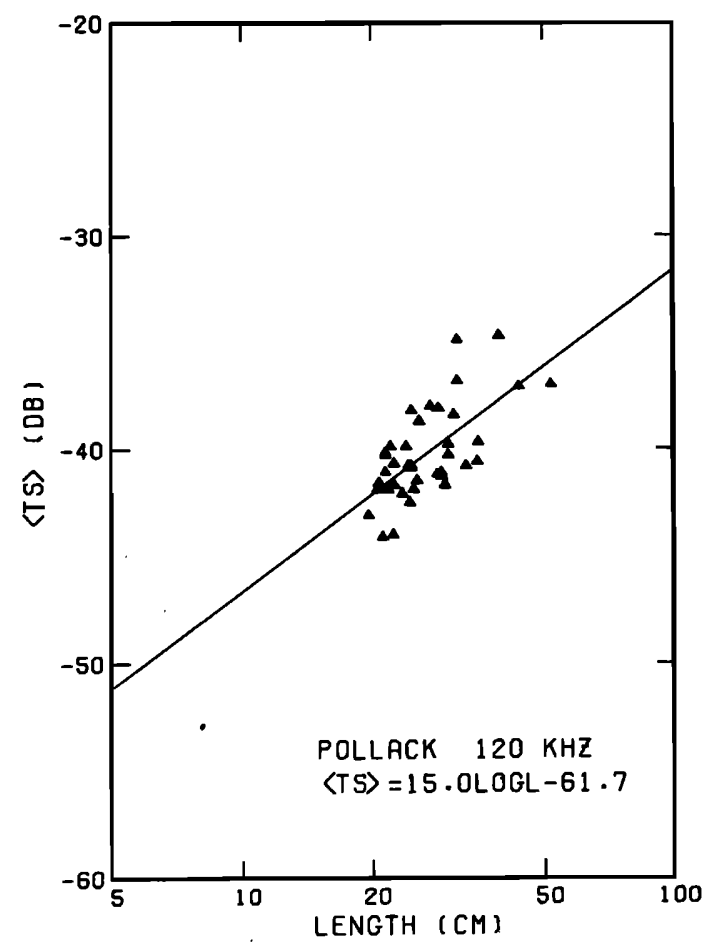

FIG. 8. Scatter diagram and regression of $\langle\mathrm{TS}\rangle-4,4,16,2.5$ on $l$ for pollack at $120 \mathrm{kHz}$.

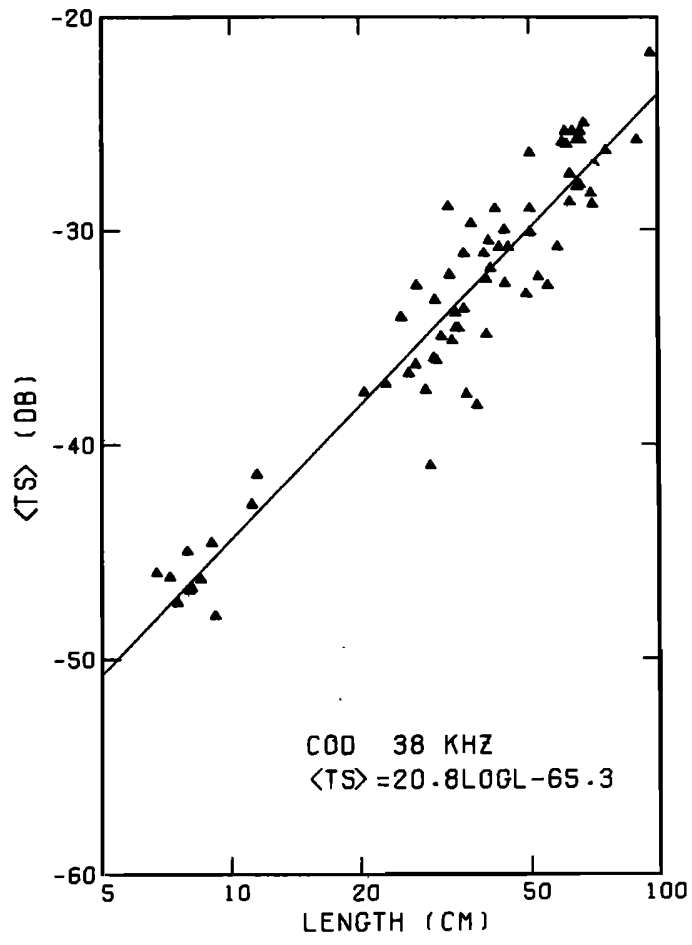

FIG. 9. Scatter diagram and regression of $\langle\mathrm{TS}\rangle_{0,5,2.5}$ on $l$ for cod at $38 \mathrm{kHz}$.

\section{RESULTS}

Scatter diagrams of $\langle\mathrm{TS}\rangle_{-4,4,16,2.5}$ for the six speciesand frequency-discriminated data sets described in $\mathrm{Ta}-$ ble I are presented together with the corresponding linear regression equations in Figs. 3-8. Scatter diagrams of $\langle\mathrm{TS}\rangle_{0,5,2.5}$ and $\langle\mathrm{TS}\rangle_{0,2,2.5}$ are similar, except

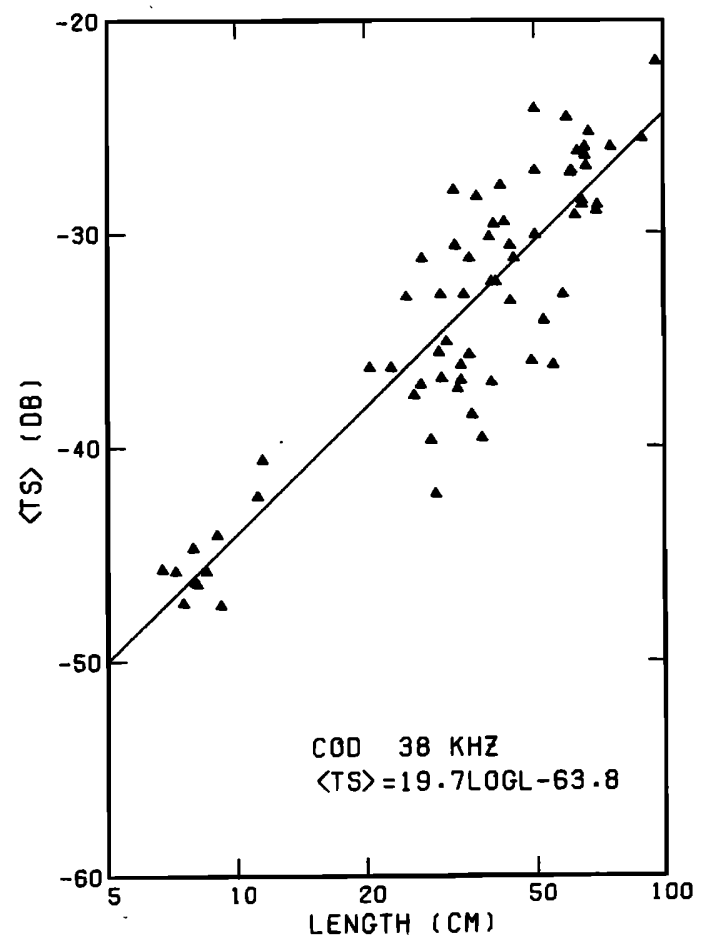

FIG. 10. Scatter diagram and regression of $\langle\mathrm{TS}\rangle_{0,2,2.5}$ on $l$ for cod at $38 \mathrm{kHz}$.

Kenneth G. Foote: Averaging of fish target strength functions 
TABLE II. Coefficients and associated statistics of linear regressions of $\langle\text { TS }\rangle_{\bar{\theta}, \sigma_{\theta}, 2.5}$ on $l$ based on measured gadoid target strength functions.

\begin{tabular}{|c|c|c|c|c|c|c|c|c|c|}
\hline $\bar{\theta}$ & $\sigma_{\theta}$ & Species & $\begin{array}{c}\text { Frequency } \\
(\mathrm{kHz})\end{array}$ & $\hat{m}$ & {$[\mathrm{SE}(\hat{m})]$} & $\hat{b}$ & {$[\operatorname{SE}(\hat{b})]$} & SE & $\rho$ \\
\hline \multirow[t]{6}{*}{-4.4} & \multirow[t]{6}{*}{16} & Cod & 38 & 21.9 & 0.6 & -68.3 & 0.9 & 1.4 & 0.979 \\
\hline & & Cod & 120 & 23.9 & 0.7 & -73.7 & 1.0 & 1.4 & 0.984 \\
\hline & & Saithe & 38 & 20.4 & 0.5 & -66.7 & 0.8 & 1.0 & 0.983 \\
\hline & & Saithe & 120 & 19.8 & 0.7 & -68.0 & 1.0 & 1.4 & 0.971 \\
\hline & & Pollack & 38 & 19.1 & 1.5 & -66.3 & 2.2 & 1.3 & 0.886 \\
\hline & & Pollack & 120 & 15.0 & 2.8 & -61.7 & 4.0 & 1.6 & 0.658 \\
\hline \multirow[t]{6}{*}{0} & \multirow[t]{6}{*}{5} & Cod & 38 & 20.8 & 0.9 & -65.3 & 1.4 & 2.2 & 0.946 \\
\hline & & Cod & 120 & 24.1 & 1.0 & -72.3 & 1.5 & 2.2 & 0.963 \\
\hline & & Saithe & 38 & 19.1 & 0.7 & -62.4 & 1.0 & 1.4 & 0.967 \\
\hline & & Saithe & 120 & 18.9 & 0.8 & -63.8 & 1.1 & 1.5 & 0.963 \\
\hline & & Pollack & 38 & 18.9 & 2.0 & -63.6 & 2.9 & 1.6 & 0.824 \\
\hline & & Pollack & 120 & 15.3 & 2.8 & -58.7 & 4.0 & 1.6 & 0.665 \\
\hline \multirow[t]{6}{*}{0} & \multirow[t]{6}{*}{2} & Cod & 38 & 19.7 & 1.2 & -63.8 & 1.9 & 3.0 & 0.894 \\
\hline & & Cod & 120 & 24.8 & 1.6 & -74.1 & 2.4 & 3.5 & 0.920 \\
\hline & & Saithe & 38 & 17.2 & 1.0 & -59.7 & 1.4 & 2.0 & 0.924 \\
\hline & & Saithe & 120 & 18.4 & 1.0 & -62.7 & 1.4 & 1.9 & 0.937 \\
\hline & & Pollack & 38 & 16.0 & 2.3 & -58.8 & 3.4 & 1.9 & 0.727 \\
\hline & & Pollack & 120 & 14.2 & 3.3 & -56.6 & 4.7 & 1.9 & 0.583 \\
\hline
\end{tabular}

for the degree of dispersion about the regression line, which is illustrated for the representative case of cod at $38 \mathrm{kHz}$ in Figs. 9 and 10 . The results of the regres sion analyses of the tilt angle distribution-, species-, and frequency-discriminated averaged data are presented in Table II.

Averaging of the squared backscattering cross sections yields similar results. The scatter diagram of $\left\langle\mathrm{TS}^{2}\right\rangle_{-4.4,16,2.5}^{1 / 2}$ for cod at $38 \mathrm{kHz}$ is shown in Fig. 11. The results of the regression analyses of the root-meansquare target strengths as discriminated by tilt angle

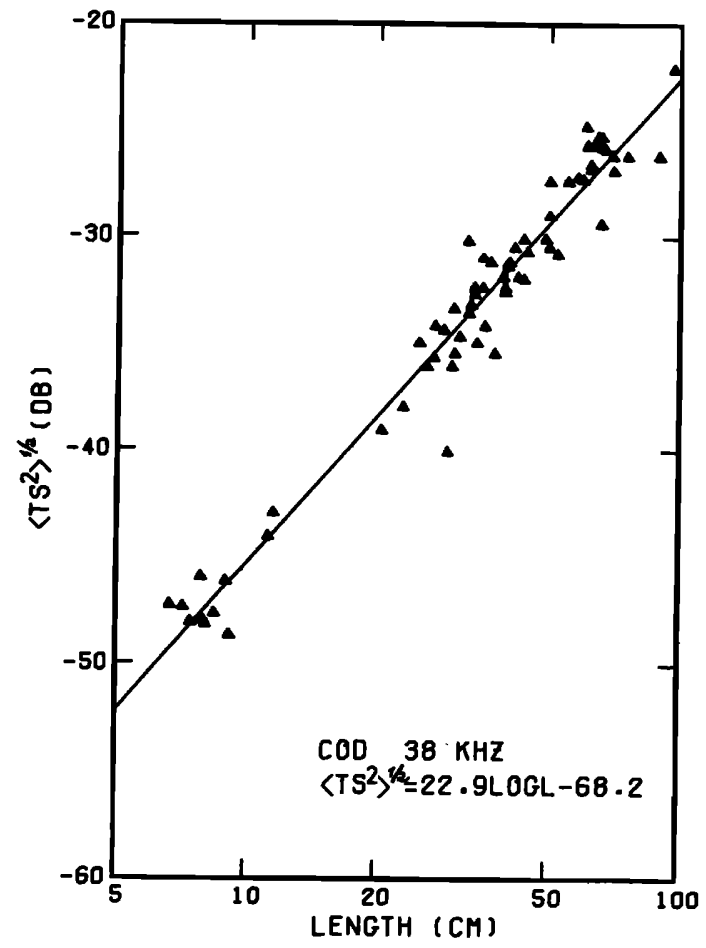

FIG. 11. Scatter diagram and regression of $\left\langle T^{2}\right\rangle_{-4,4,16,2.5}^{1 / 2}$ on $l$ for cod at $38 \mathrm{kHz}$. distribution, species and frequency are shown in Table III.

\section{DISCUSSION}

The orientation distribution common to the averaging of the measured gadoid dorsal aspect target strength functions in Figs. 3-8 is that observed by Olsen ${ }^{19}$ for free-swimming cod in the sea. It is described by an essentially normal distribution with mean and standard deviation of -4.4 and $16 \mathrm{deg}$, respectively. The same target strength functions have also been averaged with respect to two other tilt angle distributions of the same functional form, but with mean and standard deviation pairs of $(0,5)$ and $(0,2) \mathrm{deg}$. These distributions are postulated to correspond to modes of schooling behavior in which the fish are dispersed in varying degrees in tilt angle about a mean horizontal orientation. Figures 9 and 10 present examples of ave raging with respect to these two orientation distributions for the case of the measured target strength functions of cod at $38 \mathrm{kHz}$.

Insofar as the derived data of the figures are independent, follow normal distributions about mean values which lie on a straight line, and have a variance about their mean values which is independent of fish length, for which there is prima facie evidence, there is justification for the linear minimum variance regression analyses applied to the data. At least the various sets. of data are individually sufficiently homogeneous to permit their characterization by simple linear regression equations. The results of this regression analysis when applied to the data of Figs. 3-10 and to the remaining averaged target strengths not shown on scatter diagrams are summarized in Table II.

Under assumption of the three conditions stated above for application of linear minimum variance analysis, analysis of covariance may be applied to the various regressions to determine whether they are significantly 
TABLE III. Coefficients and associated statistics of linear regressions of $\left\langle\operatorname{TS}^{2}\right\rangle^{1 / 2}{ }_{\theta, \sigma_{\theta}, 2.5}$ on $l$ based on measured gadoid target strength functions.

\begin{tabular}{|c|c|c|c|c|c|c|c|c|c|}
\hline $\bar{\theta}$ & $\sigma_{\theta}$ & Species & $\begin{array}{l}\text { Frequency } \\
\quad(\mathrm{kHz})\end{array}$ & $\hat{m}$ & {$[\mathrm{SE}(\hat{m})]$} & $\hat{b}$ & {$[\operatorname{SE}(\hat{b})]$} & $\mathbf{S E}$ & $\rho$ \\
\hline \multirow[t]{6}{*}{-4.4} & \multirow[t]{6}{*}{16} & Cod & 38 & 22.9 & 0.6 & -68.2 & 0.9 & 1.5 & 0.978 \\
\hline & & Cod & 120 & 24.3 & 0.8 & -72.2 & 1.2 & 1.7 & 0.978 \\
\hline & & Saithe & 38 & 21.0 & 0.6 & -65.7 & 0.8 & 1.1 & 0.981 \\
\hline & & Saithe & 120 & 19.5 & 0.8 & -64.9 & 1.2 & 1.6 & 0.961 \\
\hline & & Pollack & 38 & 20.3 & 1.8 & -66.1 & 2.6 & 1.4 & 0.873 \\
\hline & & Pollack & 120 & 17.9 & 2.6 & -62.7 & 3.8 & 1.6 & 0.743 \\
\hline \multirow[t]{6}{*}{0} & \multirow[t]{6}{*}{5} & Cod & 38 & 21.9 & 0.8 & -66.0 & 1.3 & 2.0 & 0.958 \\
\hline & & Cod & 120 & 24.3 & 1.0 & -71.3 & 1.5 & 2.2 & 0.965 \\
\hline & & Saithe & 38 & 20.4 & 0.7 & -63.3 & 1.0 & 1.4 & 0.971 \\
\hline & & Saithe & 120 & 19.2 & 0.9 & -62.7 & 1.3 & 1.7 & 0.954 \\
\hline & & Pollack & 38 & 20.2 & 2.2 & -64.5 & 3.1 & 1.8 & 0.823 \\
\hline & & Pollack & 120 & 17.5 & 2.9 & -60.2 & 4.1 & 1.7 & 0.708 \\
\hline \multirow[t]{6}{*}{0} & \multirow[t]{6}{*}{2} & Cod & 38 & 20.4 & 1.2 & -64.2 & 1.8 & 2.9 & 0.906 \\
\hline & & Cod & 120 & 24.6 & 1.5 & -72.7 & 2.2 & 3.3 & 0.926 \\
\hline & & Saithe & 38 & 18.6 & 0.9 & -61.0 & 1.3 & 1.8 & 0.942 \\
\hline & & Saithe & 120 & 18.8 & 1.0 & -62.1 & 1.5 & 2.0 & 0.937 \\
\hline & & Pollack & 38 & 17.7 & 2.4 & -60.7 & 3.4 & 2.0 & 0.756 \\
\hline & & Pollack & 120 & 15.7 & 3.4 & -57.4 & 4.9 & 2.0 & 0.602 \\
\hline
\end{tabular}

different or can be combined, as to simplify their use. Detailed statistical analyse ${ }^{22,23}$ demonstrate that there are significant species, frequency, and orientation distribution differences among the regressions, which establishes and extends earlier more restricted observations of species differences. ${ }^{4,8,24}$ The present analysis has been undertaken for representation of the data both without wavelength normalization, as in all of the scatter diagrams and regression analyses reported here, and with the wavelength normalization first used by Love, ${ }^{25}$ and subsequently used in Refs. 26-29. The results, while different in individual cases for the two representation schemes, are indistinguishable in the whole: some regressions can be merged in either case, but generally the various regressions cannot be merged. This finding is also supported by similar analysis of the maximum dorsal aspect target strengths of the same gadoid specimens at the two ultrasonic frequencies of measurement, both without and with wavelength normalization. These maximum values, which are essentially those presented in Ref. 4 , are significantly different from their averaged counterparts presented in this study.

With regard to the high quality and general homogene- ity of the data base, the conclusion that there are significant species, frequency, and orientation distribution differences in target strength-to-fish length regressions is itself significant. The implication or penalty for misusing or misapplying target strength regressions in the acoustic estimation of fish abundance is evidently a systematic, uncontrollable bias. Because knowledge of the fish species under observation is assumed in fisheries surveys and because the frequency and other characteristics of the acoustic sensing system are fixed, the target strength-to-fish length regression should be determined for the same sonar frequency of use, fish species of observation, and orientation distribution characteristic of these fish. Under suitable conditions, such as those in which schooling fish of homogeneous composition are observed by a calibrated fisheries sonar, and then sampled by trawling, with presumed determination of schooling density, the orientation distribution could be inferred. With sufficient experience, presumably, knowledge of this acoustically observable manifestation of behavior could lessen or eliminate uncertainty over the pertinent orientation distribution. In any case, as was shown in Ref. 12, maximum dorsal aspect target strengths should not

TABLE IV. Coefficients and associated statistics of linear regressions of $\langle\mathrm{TS}\rangle_{\bar{\theta}}, \sigma_{\theta}, 2.5$ on $l$ based on Love's model for the target strength of a fish at any aspect.

\begin{tabular}{cccccccccc}
\hline \hline \multirow{\theta}{*}{$\bar{\theta}$} & & $\begin{array}{c}\text { Type of } \\
\text { description }\end{array}$ & $\begin{array}{c}\text { Frequency } \\
(\mathrm{kHz})\end{array}$ & $\hat{m}$ & {$[\mathrm{SE}(\hat{m})]$} & $\hat{b}$ & {$[\mathrm{SE}(\hat{b})]$} & $\mathrm{SE}$ & $\rho$ \\
\hline-4.4 & \multirow{2}{*}{16} & arc & 38 & 18.6 & 0.01 & -64.0 & 0.03 & 0.05 & $1.000^{-}$ \\
& & arc & 120 & 18.6 & 0.01 & -64.6 & 0.02 & 0.05 & $1.000^{-}$ \\
& & area & 38 & 18.7 & 0.01 & -63.4 & 0.03 & 0.05 & $1.000^{-}$ \\
& & area & 120 & 18.7 & 0.01 & -64.0 & 0.02 & 0.05 & $1.000^{-}$ \\
& & & & & & & & & \\
0 & 5 & arc & & & & & & & \\
& & arc & 120 & 18.8 & 0.01 & -63.1 & 0.03 & 0.05 & $1.000^{-}$ \\
& & area & 38 & 18.8 & 0.01 & -63.6 & 0.02 & 0.05 & $1.000^{-}$ \\
& & area & 120 & 18.7 & 0.01 & -63.3 & 0.02 & 0.05 & $1.000^{-}$ \\
\hline \hline
\end{tabular}


be used in place of the corresponding averaged dorsal aspect target strengths for fish observed by directional downward-looking sonars.

The fact that application of analysis of covariance to the averaged target strength regressions presented here reveals significant species and frequency differences suggests inadequacy in Love's model for the target strength function of a fish at any aspect. ${ }^{27}$ By its very nature this model ignores systematic variations in the target strength function due to species. Possible frequency and behavior or orientation distribution differences among regressions based on Love's model have been investigated by repeating the averaging computations described above, but with use of target strength functions derived from Love's model in place of measured target strength functions. For target strength functions derived from Love's description of target strengths along arcs, the averaging proceeds identically to that of the computational example. For functions derived from Love's description of target strengths over angular areas, however, the averaging process is modified to account for the roll angle dependence included in this more general description.

Results obtained by regressing the averaged target strengths based on Love's model on fish length are presented in Table IV for averaging with respect to two kinds of behavior. These results obtain for the general length ranges of interest, which are roughly 1 to 100 wavelengths, without regard to the particular length
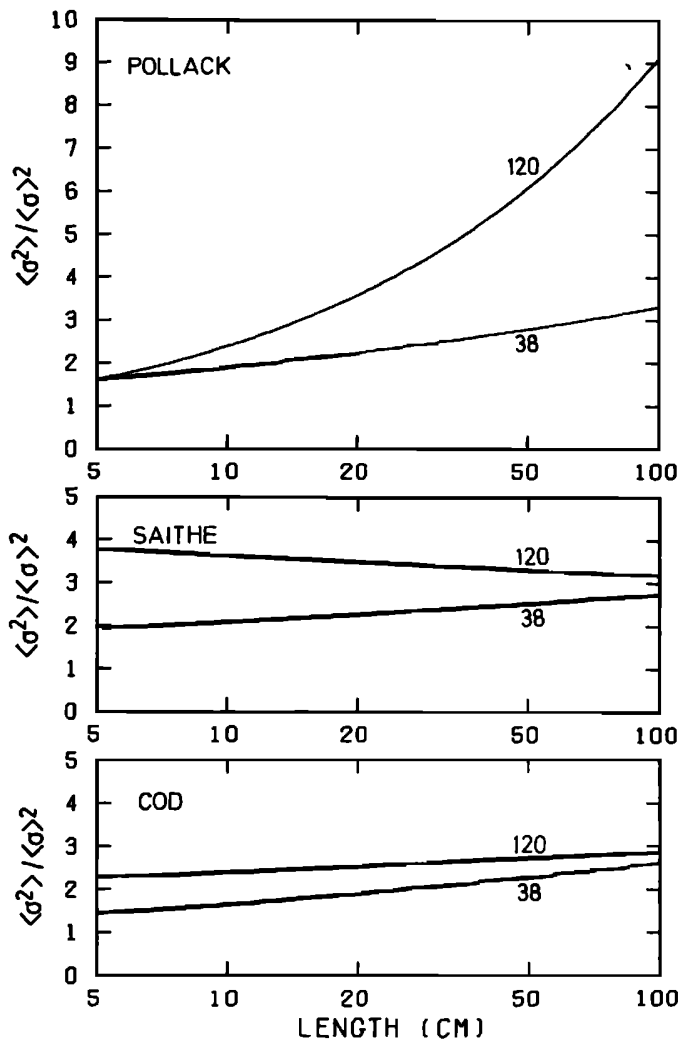

FIG. 12. Length dependence of $\left\langle\sigma^{2}\right\rangle /\langle\sigma\rangle^{2}$ in the mean for three gadoid species at two ultrasonic frequencies for averaging with respect to a tilt angle distribution with parameters $\left(\bar{\theta}, \sigma_{\theta}\right)=(-4.4,16) \mathrm{deg}$ and a sonar with half-beamwidth of 2.5 deg. Values of frequency given in $\mathrm{kHz}$.

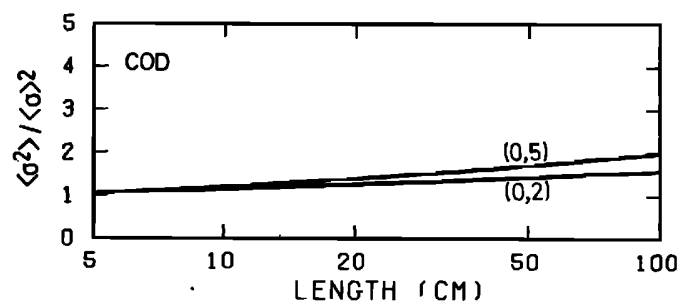

FIG. 13. Length dependence of $\left\langle\sigma^{2}\right\rangle /\langle\sigma\rangle^{2}$ in the mean for cod at $38 \mathrm{kHz}$ for averaging with respect to two tilt angle distributions, with parameters $\left(\bar{\theta}, \sigma_{\theta}\right)=(0,5) \mathrm{deg}$ and $(0,2) \mathrm{deg}$, and a sonar with half-beamwidth of $2.5 \mathrm{deg}$.

distribution chosen for the regression analysis because of the homogeneity of Love's model. This same homogeneity explains the similarity in regressions for the two kinds of behavior; differences simply express the tendency of the mean backscattering cross section to decrease with increasing sizable excursion from mean tilt angles in the vicinity of the maximum dorsal aspect target strength. The case of behavior defined by the orientation distribution with parameters $(0,2) \mathrm{deg}$ is omitted as averaged values based on this distribution are indistinguishable from thosed based on the tilt angle distribution with parameters $(0,5) \mathrm{deg}$. This is also explicable in terms of the homogeneity of the target strength function modeled by Love, which differs noticeably from measured functions by the relative coarseness of its angular description.

Frequency differences in the regressions of Table IV, which are also slight, owe their origin to the method of description of target strengths by wavelength-normalized backscattering cross sections. This method of representing the length dependence of target strengths, except for the case of an exact quadratic length dependence of backscattering cross section, imposes a frequency bias. Differences in the corresponding regressions based on arc and area descriptions are slight, but consistent: averaged target strengths computed according to the arc description are generally less than those of the area description because of the absence of generally larger dorsilateral aspect target strengths included in the second description.

It does appear reasonable that averaged target strength-to-fish length regressions based on Love's model can be used in estimating fish abundance in situations where knowledge of the observed fish is negli-

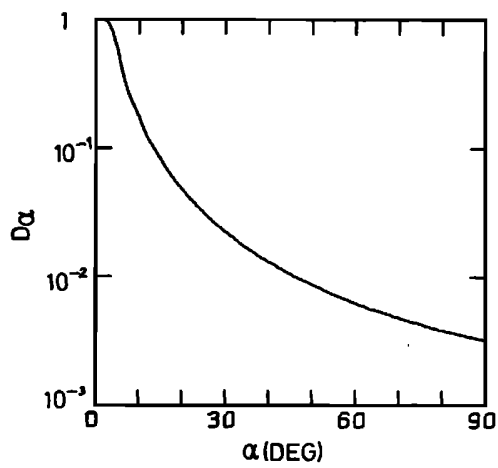

FIG. 14. Dependence of beam pattern factor $D_{\alpha}$, defined in Eq. (27), on $\alpha$. 
gible or highly uncertain. In situations in which gadoids, at least, are known to be the primary objects of observation, the use of regressions based on measured target strength functions are superior. A statistical analysis of errors incurred in the acoustic estimation of gadoid abundance by using averaged target strength-tofish length regressions based on Love's model instead of comparable regressions based on species- and frequency-discriminated data supports this conclusion.

The discussion, which has thus far been concerned with averaged target strengths, could be repeated for the root-mean-square target strengths also computed in this study. The similarity of averaged and root-meansquare target strengths, for the same target strength functions and manner of averaging, is established by comparison of comparable entries in Tables II and III. Comparison of Figs. 3 and 11 for respective averaging of the backscattering cross sections and their squares for the same measured cod data at $38 \mathrm{kHz}$ with respect to the same orientation distribution reinforces the notion of this similarity.

A detailed comparison of the respective averaged and root-mean-square target strengths does reveal differences. Some of these are described in Figs. 12 and 13, where the ratio of averaged-squared backscattering cross section and squared-averaged backscattering cross section in the mean are plotted against fish length. Restriction of the computed ratios to the approximate length ranges described in Table $I$ is understood. In terms of the root-mean-square and averaged target strengths, the computed ratio is

$$
\left\langle\sigma^{2}\right\rangle /\langle\sigma\rangle^{2}=10^{\left(\left\langle\mathrm{Ts}^{2}\right\rangle^{1 / 2}-\langle\mathrm{TS}\rangle\right) / 5} \text {, }
$$

which follows from Eqs. (22) and (23).

A quantity of greater significance in computing errors in fish abundance estimates is the ratio of observable mean-squared backscattering cross section to squaredmean backscattering cross section,

$$
\frac{\int_{0}^{\alpha} b^{4}(\theta) \sin \theta d \theta \int_{0}^{\alpha} \sin \theta d \theta}{\left(\int_{0}^{\alpha} b^{2}(\theta) \sin \theta d \theta\right)^{2}} \frac{\left\langle\sigma^{2}\right\rangle}{\left\langle\sigma^{2}\right.} \equiv D_{\alpha}^{-1} \frac{\left\langle\sigma^{2}\right\rangle}{\langle\sigma\rangle^{2}},
$$

where the beam pattern $b(\theta)$ is defined in Eq. (13), and $\alpha$ describes the angular extent of observed fish, as measured from the acoustic axis, which for the particular computational example considered here is assumed to be at least $5 \mathrm{deg}$. The factor $D_{\alpha}$ cannot exceed unity, which is seen immediately by consideration of the Schwarz inequality. The dependence of $D_{\alpha}$ on the upper limit of integration $\alpha$ is shown in Fig. 14 .

Computations of the sort described in Eqs. (26) and (27) and presented in Figs. 12-14 are important in determining errors associated with acoustic estimates of fish abundance. Earlier treatments ${ }^{1-3}$ of the contribution of the variability in target strength function to these errors have ignored the inseparable geometric effects of perspective and beam patterns. The present study may be viewed, therefore, as both revising and extending this work.

\section{SUMMARY}

The principal accomplishments of this paper are both theoretical and practical. Averaging of the target strength functions of fish has been treated in a general manner which accounts for effects due to geometry, including the effect of perspective on altering apparent or observed target strengths; behavior of observed fish through their orientation distribution; and beam patterns of observing sonar. The philosophy enunciated by Lozow and Suomala, ${ }^{1}$ that the methods of system analysis can only be applied to studies of the acoustic estimation of fish abundance by considering well-defined situations, has been accepted tacitly in consideration of a particular computational example. This example is important because of its modeling of realistic situations in which fish abundance is determined by means of directional downward-looking echo sounders operating at ultrasonic frequencies. The availability of high quality target strength data for three gadoid species at two ult rasonic frequencies has been exploited in actual computations of averaged and averaged-squared backscattering cross sections. Species, frequency, and orientation distribution differences in simple linear regressions based on the logarithmic expression of these cross sections has been noted. Comparisons of averaged and averaged-squared backscattering cross sections, to facilitate estimation of errors in fish abundance estimates, have been presented for averaged data which are homogeneous in species, frequency, and orientation distribution.

The present work may be viewed, additionally, in the language of Middleton, ${ }^{30}$ as providing some details of the basic scattering elements and their computation which are needed to describe active underwater acous tic channels at high frequencies when dominated by scattering from fish.

\section{ACKNOWLEDGMENTS}

I wish to thank M. Aksland, Department of Fisheries Biology, University of Bergen, and J. Dalen and $\mathrm{K}$. Olsen, Marine Research Institute, Bergen, Norway, for their criticism of the manuscript.

${ }^{1}$ J. B. Lozow and J. B. Suomala, Jr., "The Application of Hydroacoustic Methods for Aquatic Biomass Measurements. A note on Echo Envelope Sampling and Integration," Massachusetts Inst. Technol., Cambridge, MA, Report No. MIT SG $72-8$ (1972).

${ }^{2}$ P. H. Moose and J. E. Ehrenberg, "An expression for the variance of abundance estimates using a fish echo integrator," J. Fish. Res. Board Can. 28, 1293-1301 (1971).

${ }^{3} \mathrm{H}$. Bodholt, "Variance Error in Echo Integrator Output," in Hydro-Acoustics in Fisheries Research, edited by A. R. Margetts (Rapp. P.-V. Réun. Cons. int. Explor. Mer 170, Paris, 1977), pp. 196-204.

${ }^{4} \mathrm{O}$. Nakken and K. Olsen, "Target Strength Measurements of Fish," in Hydro-Acoustics in Fisheries Research, edited by A. R. Margetts (Rapp. P.-V. Réun. Cons. int. Explor. Mer 170, Paris, 1977), pp. 52-69.

${ }^{5}$ M. L. Peterson, C. S. Clay, and S. B. Brandt, "Acoustic estimates of fish density and scattering function," J. Acoust. Soc. Am. 60, 618-622 (1976).

${ }^{6}$ V. G. Samovol'kin, "Apparatus and experimental technique 
for the study of ultrasonic backscattering by biological objects in water, "Okeanologiya 14, 149-152 (1974).

${ }^{7} \mathrm{~V}$. G. Samovol'kin, "Acoustic backscattering cross sections of small fish, crustaceans, and medusae as functions of their foreshortening," Okeanologiya 14, 655-660 (1974).

${ }^{8} \mathrm{~K}$. I. Yudanov, "Reflection Power of Commercially Important Fishes and Fish Concentrations," in Hydro-Acoustics in Fisheries Research, edited by A. R. Margetts (Rapp. P.-V. Réun. Cons. int. Explor. Mer 170, Paris, 1977), pp. 185-188.

${ }^{9}$ K. G. Foote, "Analysis of empirical observations on the scattering of sound by encaged aggregations of fish," Fiskeridir. Skr. Ser. Havunders. 16, 423-456 (1978).

${ }^{10}$ J. E. Ehrenberg and D. W. Lytle, "Some Signal Processing Techniques for Determining the Variance in Acoustic Stock Abundance Estimates, " in Hydro-Acoustics in Fisheries Research, edited by A. R. Margetts (Rapp. P.-V. Réun. Cons. int. Explor. Mer 170, Paris, 1977), pp. 205-213.

${ }^{11} J$. E. Ehrenberg, "Two Applications for a Dual-Beam Transducer in Hydroacoustic Fish Assessment Systems, " in Proceedings of the IEEE Ocean 74 International Conference on Engineering in the Ocean Environment, Halifax, Nova Scotia, 21-23 August 1974 (IEEE, New York, 1974).

${ }^{12} \mathrm{~K}$. G. Foote, "Effect of fish bahavior on echo energy: the need for measurements of orientation distributions," J. Cons. Cons. Int. Explor. Mer. 39 (2) (1979) (in press).

${ }^{13} \mathrm{~K}$. G. Foote and O. Nakken, "Dorsal aspect target strength functions of six fishes at two ultrasonic frequencies, "Fisken Havet, Ser. B, No. 3, 1-96 (June 1978).

${ }^{14} \mathrm{~K}$. Olsen "Influence of Vessel Noise on Behavior of Herring," in "Modern Fishing Gear of the World: 3," edited by H. Kristjonsson [Fishing News (Books) Ltd., London, 1971], pp. 291-294.

${ }^{15} \mathrm{~K}$. Olsen, "Evidence for localization of sound by fish in schools," in "Sound Reception in Fish," edited by A. Schuijf and A. D. Hawkins (Elsevier, Amsterdam, 1976), pp. 257268.

${ }^{16} \mathrm{Y}$. Maniwa, "Effect of Vessel Noise in Purse Seining," in Modern Fishing Gear of the World: 3, edited by H. Kristjonsson [Fishing News (Books) Ltd., London, 1971], pp. 294296.

${ }^{17} \mathrm{H}$. Mohr, "Behavior Patterns of Different Herring Stocks in Relation to Ship and Midwater Trawl, " in Modern Fishing Gear of the World: 3, edited by $\mathrm{H}$. Kristjonsson [Fishing News (Books) Ltd., London, 1971], pp. 368-371.

${ }^{18}$ R. W. G. Haslett, "Determination of the acoustic backscat- tering patterns and cross sections of fish," Brit. J. Appl. Phys. 13, 349-357 (1962).

${ }^{19} \mathrm{~K}$. Olsen, "Orientation measurements of cod in Lofoten obtained from underwater photography and their relation to target strength," ICES CM 1971/B:17, 8 pp. (Mimeo).

${ }^{20} \mathrm{~A}$. K. Beltestad, "Feeding, vertical migration, and schooling of O-group herring (Clupea havengus $L$.) in relation to light intensity," dissertation, University of Bergen, Bergen, Norway (1974) (in Norwegian).

${ }^{21} \mathrm{~K}$. G. Foote, "Theoretical estimation of the mean echo intensity-fish number density relation for encaged saithe in the dorsal aspect, " Fiskeridir. Skr. Ser. Havunders. 16, 457464 (1978).

${ }^{22} \mathrm{~K}$. G. Foote, "Representations of length dependence of acoustic target strengths of fish, "J. Fish. Res. Board Can. 36, 1490-1496 (1979).

${ }^{23}$ K. G. Foote, "Fish target strength-to-length regressions for application in fisheries research," in Ultrasonics International 79 Conference Proceedings, Graz, Austria, 1518 May 1979 (IPC Sci. Tech. Press, London, 1979), pp. 327332.

${ }^{24}$ K. I. Yudanov, A. A. Gan'kov, and O. E. Shatoba, "Reflecing Properties of Commercial Fishes of the Northern Basin," Rybn. Khoz. (Moscow) 42(12), 57-60 (1966) (in Russian).

${ }^{25} \mathrm{R}$. H. Love, "Maximum Side-Aspect Target Strength of an Individual Fish," J. Acoust. Soc. Am. 46, 746-752 (1969).

${ }^{26} \mathrm{R}$. H. Love, "Dorsal-Aspect Target Strength of an Individual Fish," J. Acoust. Soc. Am. 49, 816-823 (1971).

${ }^{27}$ R. H. Love, "Target strength of an individual fish at any aspect," J. Acoust. Soc. Am. 62, 1397-1403 (1977).

${ }^{28}$ B. S. McCartney and A. R. Stubbs, "Measurements of the Acoustic Target Strengths of Fish in Dorsal Aspect, Including Swimbladder Resonance," J. Sound Vib. 15, 397-420 (1971).

${ }^{29}$ G. C. Goddard and V. G. Welsby, "Statistical Measurements of the Acoustic Target Strength of Live Fish, " Department of Electronic and Electrical Engineering, University of Birmingham, Birmingham, England, Departmental Memorandum No. 456 (1975).

${ }^{30} \mathrm{D}$. Middleton, "Characterization of Active underwater Acoustic Channels: Part I. A New Formulation of Scattering by Random Media and Interfaces," Applied Research Laboratories, The University of Texas at Austin, Austin, Texas, Report No. ARL-TR-74-61 (1974). 